\title{
Low-temperature scanning tunneling microscopy and spectroscopy of spatial oscillations in the density of states near domain boundaries at the $\mathrm{Ge}(111) 2 \times 1$ surface
}

\author{
D. A. Muzychenko, S. V. Savinov, V. N. Mantsevich, N. S. Maslova, and V. I. Panov \\ Faculty of Physics, Moscow State University, 119991 Moscow, Russia
}

\author{
K. Schouteden and C. Van Haesendonck \\ Laboratory of Solid-State Physics and Magnetism, Institute for Nanoscale Physics and Chemistry (INPAC), BE-3001 Leuven, Belgium
}

(Received 9 July 2009; revised manuscript received 20 November 2009; published 11 January 2010)

\begin{abstract}
By means of low-temperature scanning tunneling microscopy and scanning tunneling spectroscopy we demonstrate the existence of spatial oscillations in the local electron density of states of clean $\operatorname{Ge}(111) 2 \times 1$ surfaces. The oscillations appear exclusively in the vicinity of boundaries between domains with different atomic arrangements and are present only within a limited range of tunneling voltages approximately between 0.2 and $0.8 \mathrm{~V}$. From the spectroscopy measurements we are able to extract the energy versus wave-vector dispersion relation of the spatial oscillations in the local electron density of states. Relying on a tight-binding based model, we are able to link the observed phenomena to two-dimensional Tamm surface states that are formed within the semiconductor band gap and that are scattered at domain boundaries where translational symmetry is broken.
\end{abstract}

DOI: 10.1103/PhysRevB.81.035313

PACS number(s): 68.35.Dv, 68.37.Ef, 73.20.At

\section{INTRODUCTION}

Electronic properties of the surfaces of the elemental semiconductors $\mathrm{Si}$ and Ge have attracted a lot of interest due to their technological relevance as well as due to their fundamental scientific importance. In particular, the (111) surface has been intensively investigated since it corresponds to the natural cleavage plane. Following cleavage at room temperature both the $\mathrm{Ge}(111)$ and the $\mathrm{Si}(111)$ surface reveal the typical $2 \times 1$ reconstruction that consists of $\pi$-bonded chains of $\mathrm{Ge}$ or $\mathrm{Si}$ atoms ${ }^{1,2}$ running in the $[01 \overline{1}]$ direction. Atomically resolved scanning tunneling microscopy (STM) and scanning tunneling spectroscopy (STS) are ideal tools to investigate the details of the reconstruction and reveal possible differences between the reconstructed $\mathrm{Ge}(111)$ and $\mathrm{Si}(111)$ surfaces. $^{3-5}$ Here, we focus on the $2 \times 1$ reconstructed $\mathrm{Ge}(111)$ surface. Only every other (upper) chain of the $\pi$-bonded chains is imaged by STM, ${ }^{6}$ which can be linked to the buckling of the surface. The buckling can result in two different isomers, ${ }^{7,8}$ that are referred to as "left" (or "positive") and "right" (or "negative") buckling. A combination of experimental STM images with model-based simulations indicates that the $\mathrm{Ge}(111) 2 \times 1$ reconstruction is dominated by the right buckling. ${ }^{9}$

From STM images of the $\mathrm{Ge}(111) 2 \times 1$ surface it can be concluded that this surface consists of various domains that have a different atomic arrangement. ${ }^{10}$ Two distinct types of domain boundaries (DBs) have been observed. The first type of DB, an "antiphase" DB, which according to Ref. 10 we refer hereafter to as a type-B DB, originates from a shift of the $\pi$-bonded chains at the $\mathrm{DB}$ in the [211] direction by one half of the $2 \times$ period. For the second type of DB, the type-A $\mathrm{DB},{ }^{10}$ the atomic rows at both sides of the DB are rotated over an angle $\pi / 3$ with respect to each other. At this point it remains unclear whether different isomers are involved in the domain formation.

In 1980s, both photoemission studies ${ }^{11}$ of heavily doped Ge single crystals and theoretical calculations ${ }^{12}$ have already revealed the existence of separate occupied and nonoccupied bands for the $\mathrm{Ge}(111)$ surface electrons. More recently, the $\mathrm{Ge}(111)$ surface band gap has been determined by means of STM and STS. ${ }^{6}$ Until now, however, there have been no direct observations of effects related to the nonoccupied surface states band of the Ge(111) $2 \times 1$ surface.

Here, we report on our detailed investigation of the nonoccupied surface states on in situ cleaved Ge(111) surfaces by means of low-temperature STM and STS under ultrahigh vacuum (UHV) conditions. The presence of the nonoccupied surface states is observed to induce spatial oscillations in the local density of states (LDOS) in the vicinity of DBs on the reconstructed $\mathrm{Ge}(111) 2 \times 1$ surface. So far, such oscillations have been observed on $\mathrm{Si}(001) 2 \times 1$ and $\mathrm{Ge}(001) 2 \times 1$ surfaces only. ${ }^{13,14}$ We are able to determine the energy versus wave-vector dispersion relation of the spatial LDOS oscillations on the Ge(111) $2 \times 1$ surface. Relying on a tight-binding based model we are able to explain the observed oscillations in terms of two-dimensional (2D) Tamm surface states that are formed within the semiconductor band gap and are scattered at the DBs.

\section{EXPERIMENTAL}

STM and STS measurements were performed with a commercially available low-temperature UHV setup (Omicron Nanotechnology), consisting of a room-temperature sample preparation chamber and a low-temperature STM measurement chamber $\left(T_{\text {sample }} \simeq 4.5 \mathrm{~K}\right)$. The whole UHV system is decoupled from the building by a specially designed vibration isolation floor for optimal measurement stability. Electrochemically etched tungsten tips were used in all experiments. The tips were cleaned in situ by repeated flashing well above $1000 \mathrm{~K}$ in order to remove the surface oxide layer and additional contamination. The tip quality was routinely checked by acquiring atomic-resolution images of the "herringbone" reconstruction of the $\mathrm{Au}(111)$ surface. ${ }^{15}$ STM to- 

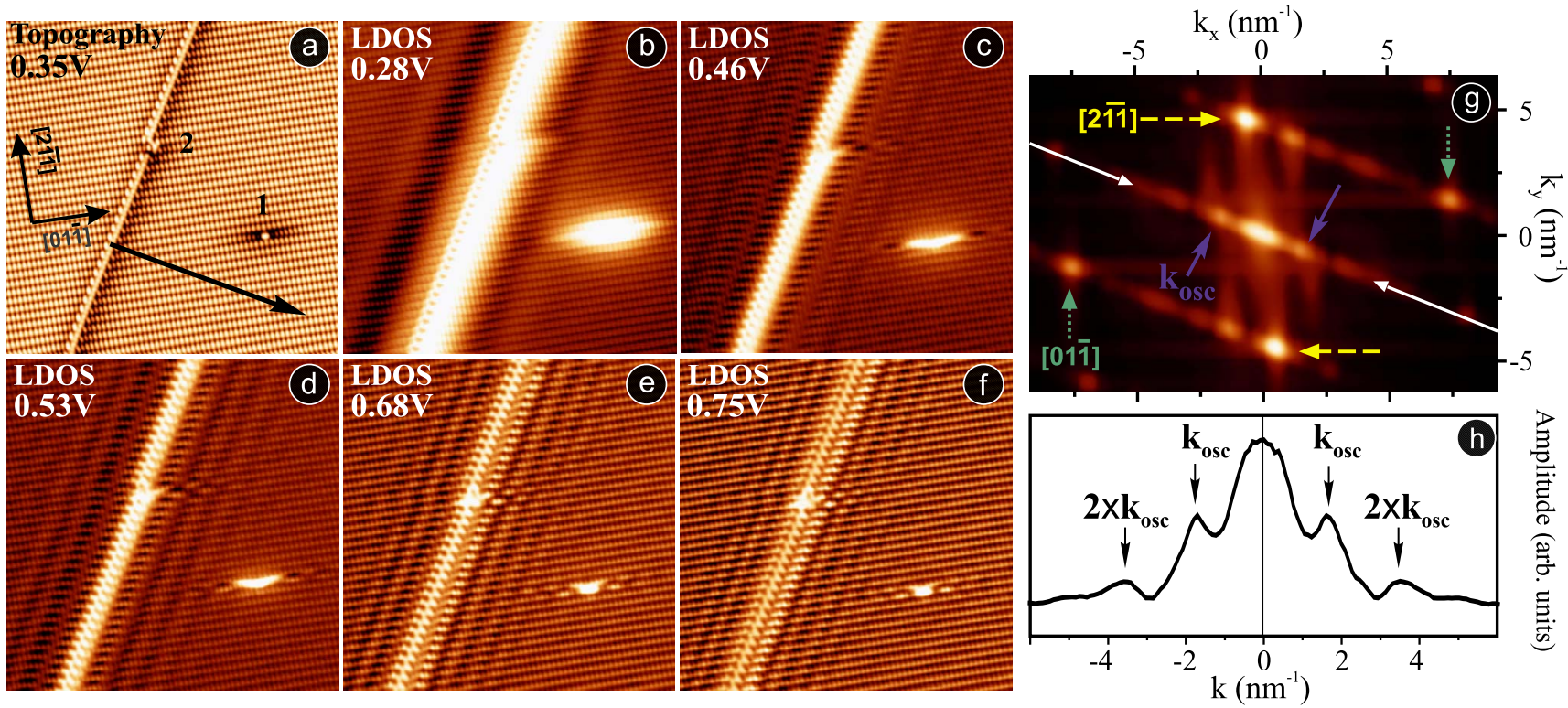

FIG. 1. (Color online) (a) $27.5 \times 27.5 \mathrm{~nm}^{2}$ constant current (tunneling current $\left.I=400 \mathrm{pA}\right) \mathrm{STM}$ image of the Ge(111) $2 \times 1$ surface. The black labels 1 and 2 indicate the location of a local defect. [(b)-(f)] Corresponding LDOS maps recorded at the indicated tunneling voltage $V$ and at a tunneling current $I=400 \mathrm{pA}$, revealing the local oscillating nature of the LDOS near a type-B DB. The wave fronts of the LDOS oscillations are parallel to the DB. (g) 2 D Fourier transform of the LDOS map in (e), revealing clear maxima corresponding to the $2 \times 1$ surface reconstruction [indicated by the dotted grayscale (green online) and dashed grayscale (yellow online) arrows] and the spatial LDOS oscillations near the type-B DB [indicated by the solid grayscale (blue online) arrows]. (h) Cross section of the Fourier transform image taken along the line in between the two white arrows in $(\mathrm{g})$.

pographic imaging was performed in constant current mode. Differential conductance images, referred to as maps of the LDOS hereafter, are acquired at selected values of the tunneling voltage $V$ with closed feedback loop by means of harmonic detection with a lock-in amplifier at modulation frequencies in the 3-7 kHz range and with modulation amplitudes in the $10-50 \mathrm{mV}$ range. The thus recorded spatial variations correspond to a map of the LDOS at an energy eV (determined by the applied tunneling voltage $V$ ) with respect to the Fermi energy. ${ }^{16}$ For these lock-in detection based STS measurements we strongly amplify the very small relative changes in the differential conductance $d I / d V(V)$. Alternatively, $I(V)$ curves were recorded with open feedback loop in the current imaging tunneling spectroscopy (CITS) mode ${ }^{16}$ with a grid size of $134 \times 134$ points, from which $d I / d V(V)$ curves are obtained numerically. From these grids maps of the local density of states can therefore be obtained as well, yielding maps that consist of $134 \times 134 d I / d V$ data points at selected values of the tunneling voltage $V$. These maps are referred to as CITS LDOS maps hereafter. In contrast to the LDOS measurements, the CITS measurements directly provide absolute numbers for the $d I / d V(V)$ data. On the other hand, the CITS measurements provide less spatial contrast when compared to the lock-in amplifier based LDOS measurements. In the text and in the figure captions tunneling voltages are always given with respect to the sample while the STM tip is virtually grounded. Image processing was performed by Nanotec WSxM. ${ }^{17}$

$5 \times 3 \times 1 \mathrm{~mm}^{3}$ Ge bars with their long axis aligned with the (111) direction were cleaved in situ at room temperature in the sample preparation chamber at a pressure of around $5 \times 10^{-11}$ mbar. The investigated Ge samples are doped with
Ga at a doping level of $2 \times 10^{16} \mathrm{~cm}^{-3}$, resulting in $p$-type bulk conductivity. The freshly cleaved samples were transferred within about $5 \mathrm{~min}$ to the STM measurement chamber. The pressure in the STM measurement chamber was about $4 \times 10^{-12}$ mbar during the STM and STS measurements. Under these conditions the cleaved Ge surfaces were observed to retain their cleanliness for 5-7 days.

\section{RESULTS}

\section{A. Observation of spatial LDOS oscillations}

Our STM experiments confirm the presence of both type-A and type-B DBs. ${ }^{10}$ However, we find that the type-A DBs occur less frequently: Only about $5 \%$ of the observed DBs are of this type. Our STS results indicate that the difference in geometry of the two types of DBs results in a pronounced difference in their electronic structure. Here, we will focus on the STM and STS results that we obtained for the type-B DBs.

In Fig. 1(a) we present a typical topographical constantcurrent STM image of a type-B DB on the clean $\mathrm{Ge}(111) 2 \times 1$ surface, together with a series of LDOS maps recorded at the indicated tunneling voltages [Figs. 1(b)-1(f)]. The topographic image [Fig. 1(a)] shows that the freshly cleaved Ge surface is atomically flat (root-mean-square roughness less than $0.1 \AA$ ), except for the DB area. The series of LDOS maps [Figs. 1(b)-1(f)] reveals the presence of pronounced spatial oscillations, extending up to several nanometers away from the DBs. The wave fronts of these spatial LDOS oscillations clearly are aligned with the DB. Consequently, the wave vector of the oscillations is perpen- 
dicular to the DB and does not coincide with the [011] direction of the $\pi$-bonded chains. This differs from previous observations on $\mathrm{Ge}(001) 2 \times 1$ and $\mathrm{Si}(001) 2 \times 1$ surfaces. On the latter surfaces the wave vector of the spatial oscillations is parallel to the $\pi$-bonded chains that are perpendicular to the DBs. ${ }^{13,14}$

The spatial LDOS oscillations on the Ge(111) $2 \times 1$ surface are visible only for a limited range of positive tunneling voltages, approximately between 0.2 and $0.8 \mathrm{~V}$. On the other hand, the wavelength of the LDOS oscillations decreases with increasing tunneling voltage. Furthermore, it can be seen in the LDOS maps that there occurs a pronounced influence of the $\mathrm{DB}$ on the electronic structure of the $\mathrm{Ge}(111) 2 \times 1$ surface. While in the topographic STM image [Fig. 1(a)] the width of the DB is observed to be between 1.5 and $1.8 \mathrm{~nm}$, this width becomes, depending on the tunneling voltage, 2-3 times larger in the LDOS images, i.e. between 3 and $6 \mathrm{~nm}$ [see Figs. 1(b)-1(f)].

In the LDOS maps it can also be observed that an individual atomic size defect, which we identified as an individual Ga impurity related defect, locally has a strong influence on the spatial oscillation pattern [labeled 1 in Fig. 1(a)]. In a small region consisting of one or two atomic chains around the atomic size defect the LDOS reveals an oscillation patterns with a wave vector that runs along the $\pi$-bonded chains and not along the DB. The statistical distribution (separation and depth) of this type of defects, which we identify as $\mathrm{Ga}$ impurity related, is consistent with the known dopant concentration for our Ge material. Apart from dopant impurities our extensive STM and STS data reveal the presence of impurity-adatom complexes. We found that the attraction of an adatom by a charged subsurface impurity is typical for the $\mathrm{Ge}(111) 2 \times 1$ surface. A more detailed analysis of the appearance of the defects related to a $\mathrm{Ga}$ impurity or a Ga impurity-adatom complex as well as the LDOS around such defects will be published elsewhere. ${ }^{18}$ The DB-induced spatial oscillations of the LDOS can be locally disturbed by a structural defect as well [labeled 2 in Fig. 1(a)] and the resulting one-dimensional (1D) local LDOS oscillations again occur along the $\pi$-bonded chains. We already reported before on the occurrence of 1D LDOS oscillations on the $\mathrm{Ge}(111) 2 \times 1$ surface. $^{19}$

In Fig. $1(\mathrm{~g})$ we present the 2D Fourier transform of the LDOS image in Fig. 1(e). We can clearly distinguish the maxima originating from the $2 \times 1$ surface reconstruction in both the $[01 \overline{1}]$ [indicated by the dotted grayscale (green online) arrows] and the [211] direction [indicated by the dashed grayscale (yellow online) arrows]. Moreover, there appear pronounced maxima due to the spatial LDOS oscillations with a wave vector $\vec{k}_{\text {osc }}$ that makes an angle of $30^{\circ}$ with the [011] direction [indicated by the solid grayscale (blue online) arrows]. The additional maxima adjacent to the [211] direction maxima are due to the periodicity of the atomic lattice in directions making a (small) angle with the [2 $\overline{11}]$ direction. In Fig. 1(h) we present a cross section of the Fourier-transform image taken along the line in between the two white arrows in Fig. 1(g). Maxima related to the spatial LDOS oscillations are clearly visible [see the arrows in Fig. $1(\mathrm{~g})]$. For the given value of the tunneling voltage

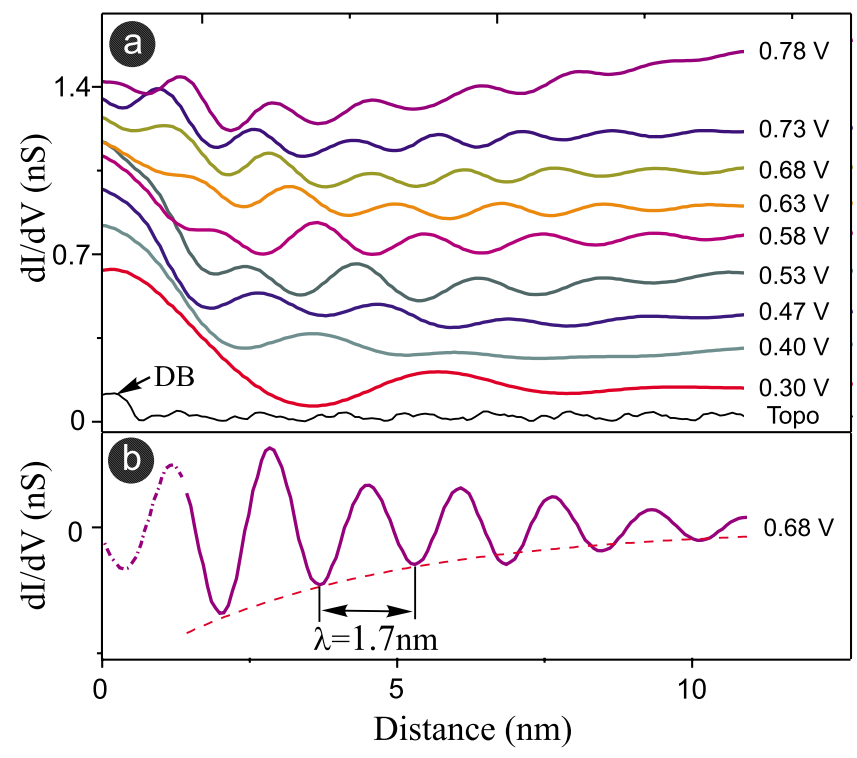

FIG. 2. (Color online) (a) LDOS height profiles taken from Figs. 1(b)-1(f) along the black arrow in Fig. 1(a). The average $d I / d V(V)$ background has been removed from each of the curves and the curves are shifted vertically for clarity. The tunneling voltage $V$ is indicated for each of the curves. (b) LDOS height profile at $V$ $=0.68 \mathrm{~V}$ with the low-frequency background filtered out. The dashed line corresponds to a fitting of the decaying oscillation amplitude by the function $A \exp (-2 x / L) \sin (2 \pi x / \lambda+\delta)$.

$V=0.68 \mathrm{~V}$, the magnitude of the wave vector $\vec{k}_{\text {osc }}$ is $1.85 \mathrm{~nm}^{-1}$. Relying on the 2D Fourier transform, it is possible to "filter" the atomic corrugation contribution out of the LDOS maps. This allows for an accurate analysis of the contribution of the spatial oscillations to the LDOS map exclusively, as will be discussed below.

\section{B. Decay of the spatial LDOS oscillations}

In this section we focus on the decay of the spatial LDOS oscillations with increasing distance from the DB. We will restrict our analysis of this decay to the type-B DB shown in Fig. 1(a). In order to draw reliable conclusions about the decay of the oscillations, an appropriate "starting point" must be chosen for the oscillation patterns. It is reasonable to define this starting point at the maximum height of the DB in the topographic constant-current STM image. The atomic corrugation is "filtered out" of the LDOS maps by relying on the Fourier-transform-based procedure described above, allowing us to isolate the contribution of the spatial oscillations to the LDOS maps. Figure 2(a) presents LDOS height profiles obtained from Figs. 1(b)-1(f) along the black arrow in Fig. 1(a). The tunneling voltage $V$ is indicated for each of the curves. The average $d I / d V(V)$ signal has been removed from the LDOS height profiles (this signal can be easily inferred from Fig. 4, see Sec. III D) and the LDOS height profiles are shifted vertically for clarity. It is clear from Fig. 2(a) that the LDOS height profiles can be described by an oscillating function with an amplitude that decreases with increasing distance from the DB, superimposed on a smoothly varying background. This behavior is most clearly 

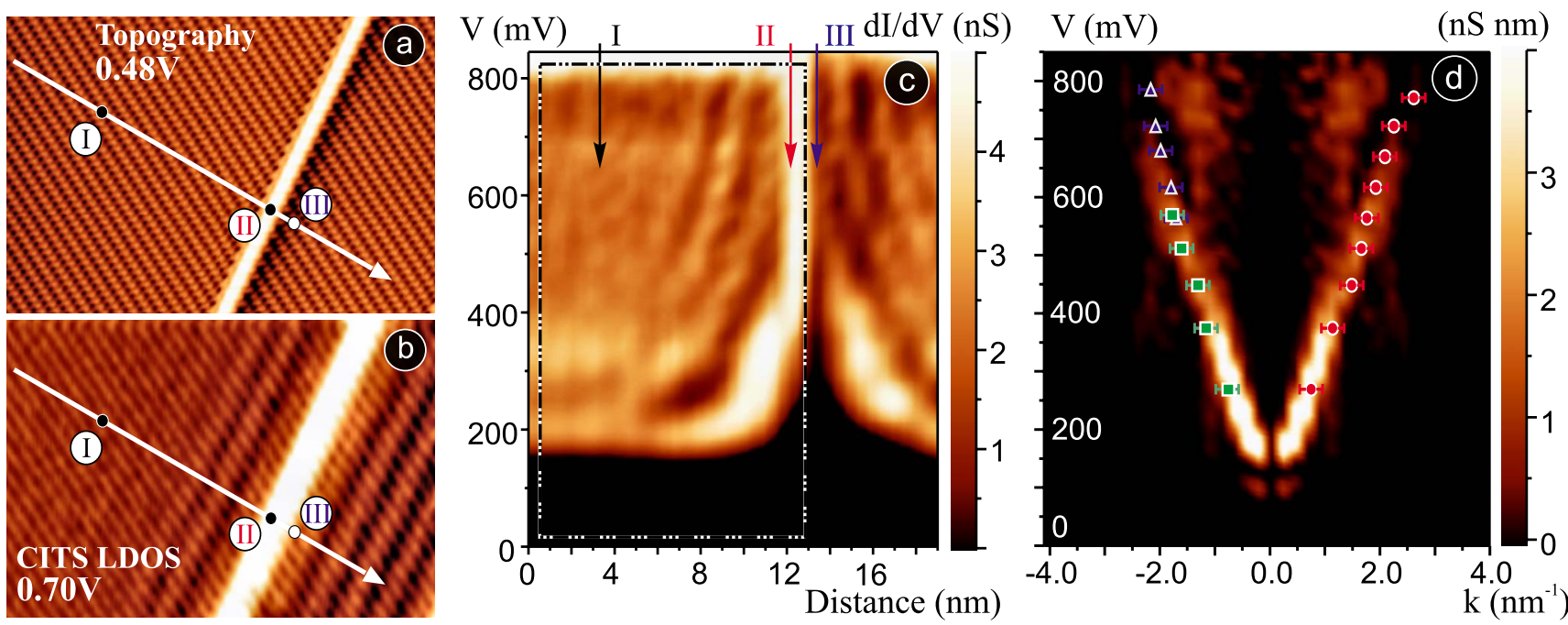

FIG. 3. (Color online) (a) $24.3 \times 16.8 \mathrm{~nm}^{2}$ constant current (tunneling current $I=400 \mathrm{pA}$ ) STM image and (b) corresponding CITS LDOS map of a type-B DB on the $\mathrm{Ge}(111) 2 \times 1$ surface obtained at the indicated tunneling voltage $V$. (c) $2 \mathrm{D}$ visualization of the $d I / d V(V)$ curves calculated from the CITS data along the white arrow in (a) and (b). The vertical cross sections indicated by the arrows labeled I-III correspond to the $d I / d V(V)$ curves at the similarly labeled locations in (a) and (b). (d) The 2D Fourier-transform image of the data enclosed by the dash-dotted rectangle in (c) provides the energy versus wave-vector dispersion behavior of the spatial LDOS oscillations. The additional data points (blue triangle, red circle, and green square symbols with error bars) are inferred from LDOS height profiles (similar to those in Fig. 2) for three different DBs.

visible for the middle profiles in Fig. 2(a). The curvature of the smoothly varying background increases with increasing tunneling voltage.

In order to unambiguously determine the wavelength of the decaying LDOS oscillations, the low-frequency background must be subtracted from the LDOS height profiles. Figure 2(b) shows the result for the profile taken at $V=0.68 \mathrm{~V}$, from which a wavelength of $1.7 \mathrm{~nm}$ can be inferred. In units of the $2 \times / 1 \times$ period of the $\mathrm{Ge}(111) 2 \times 1$ reconstruction with a unit cell of $0.69 \times 0.40 \mathrm{~nm}^{2}$ this wavelength corresponds to $2.44 / 4.23$. The wavelength is comparable to the periodicity of the atomic corrugation, confirming that it is indeed necessary to filter out the atomic corrugation of the LDOS map before taking the LDOS height profiles. The best fit of the decay of the oscillation amplitude in Fig. 2(b) turns out to be provided by an exponentially decaying sine function: $A \exp (-2 x / L) \sin (2 \pi x / \lambda+\delta) . \delta$ represents the phase shift with respect to the starting point. The decay length $L$ that provides the best fit (least-squares fitting) is 8.7 $\mathrm{nm}$. The exponential decay turned out to be superior over more simple dependencies including $1 / x$ or $1 / x^{2}$. As already discussed above, the LDOS becomes highly disturbed at the DB [see Fig. 1(b)]. The spatial extent of this disturbance can be as large as $6 \mathrm{~nm}$, depending on the applied tunneling voltage. Therefore, we did not include the first period of the LDOS oscillation [indicated by the dash-dotted part of the cross section in Fig. 2(b)] in our fitting analysis. This implies that we cannot draw any reliable conclusions about the value of the phase angle $\delta$. We note that the decay of the LDOS oscillations can be affected by thermal broadening as well as by the finite amplitude of the voltage modulation that is needed for the lock-in detection based LDOS measurements. At the end of Sec. III C we will demonstrate why the influence of the finite temperature and modulation voltage amplitude can be neglected for our measurements.
While we focus in this paper on our observation of spatial LDOS oscillations in the vicinity of type-B DBs on the Ge(111) $2 \times 1$ surface of freshly cleaved $p$-type bulk Ge, we want to stress that similar oscillations are present as well for the type-A DBs, again only within a limited range of positive tunneling voltages. The wavelength was found to be the same for both types of DBs. In both cases the wave vector of the oscillations is perpendicular to the DB and the decay of the oscillations with distance from the DB can be best described by an exponentially decaying sinusoidal function. Around a local lattice defect or around a local dopant impurity related disturbance [zero-dimensional (OD) defects] the wave vector of the spatial LDOS oscillations runs along the

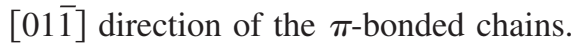

\section{Dispersion relation}

Relying on CITS measurements ${ }^{20,21}$ we were able to extract the energy versus wave-vector dispersion relation of the observed spatial LDOS oscillations. In Fig. 3(a) we present a constant current STM image of a type-B DB at the Ge(111) $2 \times 1$ surface, recorded simultaneously during the CITS measurements as described in Sec. II. Figure 3(b) is a CITS LDOS map at a tunneling voltage $V=0.7 \mathrm{~V}$. It is clear that also the CITS measurements directly reflect the presence of the spatial LDOS oscillations around a DB on the Ge(111) $2 \times 1$ surface. Figure 3(c) presents a 2D visualization of the $d I / d V(V)$ curves calculated from the CITS measurements along the white arrow in Figs. 3(a) and 3(b). The vertical arrows with labels I, II, and III indicate the position of the vertical cross sections that correspond to the $d I / d V(V)$ curves at the similarly labeled locations in Figs. 3(a) and 3(b). Figure 3(c) nicely illustrates the variation in the spatial LDOS oscillations around the DB with increasing tunneling 
voltage $V$. Here as well, the oscillations can be observed only for tunneling voltages ranging approximately between $V=+0.2 \mathrm{~V}$ and $V=+0.8 \mathrm{~V}$. Below the lower limit, which we refer hereafter to in terms of a threshold energy $E_{o n}$, there are no states available for tunneling, while above the upper limit the coupling to the underlying bulk states results in a smearing of the LDOS oscillations stemming from the surface electron states.

According to the simple model presented in Ref. 10 a type-B DB should be perfectly symmetric. From both Figs. 1(b)-1(f) and Figs. 3(b) and 3(c) it is obvious that the DBs have an asymmetric electronic structure. In Fig. 3(c) it appears that the DB is accompanied by an "energy barrier" (see the black spikelike feature on the bottom-right side of the color map). Therefore, the question arises whether the type-B DB is indeed formed in between surface areas that consist of the same isomers, ${ }^{7}$ or rather in between surface areas that consist of domains with right (negative) and left (positive) isomers. While it has been demonstrated that the unperturbed Ge(111) $2 \times 1$ surface has a right (negative) buckling, ${ }^{9}$ there does not exist any direct experimental information concerning the occurrence of the different isomers near a DB.

By performing a row by row 1D Fourier transform of the data enclosed by the dash-dotted rectangle in Fig. 3(c), the energy versus wave-vector dispersion relation of the spatial LDOS oscillations can be obtained. The result of this Fourier transform is presented in Fig. 3(d). The extra data points (blue triangle, red circle, and green square symbols with error bars), which are presented as well in Fig. 3(d), are inferred from LDOS height profiles for three different type-B DBs, similar to the ones presented in Fig. 2. It is clear that there is an excellent agreement with the dispersion derived from the CITS results.

The decay length $L$ of the LDOS oscillations is proportional to $d E / d k$, which can be estimated from Fig. 3(d), yielding $d E / d k \simeq 1 / 3 \mathrm{eV} \mathrm{nm}$. Following the procedure used by Takagi et al., ${ }^{22}$ the decay length related to the voltage modulation for the lock-in detection is $(d E / d k) / e V_{M}$, with $V_{M}$ being the modulation amplitude, leading to a decay length of about $40 \mathrm{~nm}$ for $V_{M}=10 \mathrm{meV}$. The decay length related to thermal broadening can be estimated from the Fermi-Dirac distribution function and is equal to $2.7(d E / d k) / 2 \pi k_{B} T$, resulting in a decay length of more then $600 \mathrm{~nm}$ at $T=5 \mathrm{~K}$. Both values are considerably larger than the decay length $L=8.7 \mathrm{~nm}$ extracted from the LDOS oscillation patterns (see Sec. III B).

\section{Electronic surface band structure}

Figure 4 presents three $d I / d V(V)$ spectra calculated from the CITS results for the accordingly labeled locations in Fig. 3 (a). These spectra were also included in the 2D color map in Fig. 3(b). Spectrum I corresponds to a location about $6 \mathrm{~nm}$ away from the DB where spatial LDOS oscillations can still be observed while spectra II and III are recorded on different parts of the type-B DB. The general behavior of the three $d I / d V(V)$ spectra is similar. Local maxima and minima in spectrum I correspond to the maxima and minima that appear in Fig. 3(c) along the cross sections indicated by the vertical

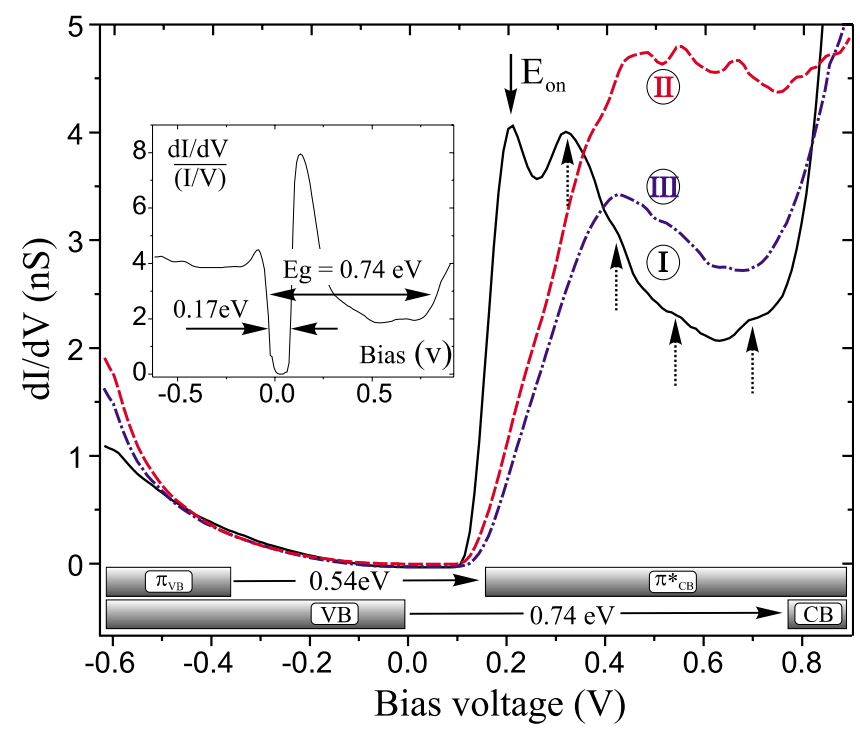

FIG. 4. (Color online) $d I / d V(V)$ spectra at the three correspondingly labeled locations on the $\mathrm{Ge}(111) 2 \times 1$ surface in Fig. 3(a). For curve I the black arrows indicate the positions of local maxima related the spatial LDOS oscillations. $E_{\text {on }}$ indicates the onset energy above which spatial LDOS oscillations are observed. Inset: normalized spectrum recorded on a defect-free area of the $\mathrm{Ge}(111) 2 \times 1$ surface. The wide gap corresponds to the bulk band-gap projected onto the $\mathrm{Ge}(111) 2 \times 1$ surface while the narrow gap corresponds to the band gap between the top of the (occupied) bulk VB and the bottom of the (unoccupied) surface-states band $\pi_{\mathrm{CB}}^{*}$.

arrows and are related to the spatial LDOS oscillations. For clarity the peak positions for spectrum I are indicated by dotted arrows. Some of these local maxima and minima appear as well in the $d I / d V(V)$ spectrum II. The spectra reveal that at more elevated energies (approximately between 0.4 and $0.8 \mathrm{eV}$ ) the DB has a higher density of states when compared to the surrounding $\mathrm{Ge}(111) 2 \times 1$ surface. Moreover, from Fig. 4 we conclude that this LDOS enhancement becomes more pronounced when approaching the center of the DB.

In the inset in Fig. 4 we present a normalized $d I / d V(V)(I / V)$ spectrum obtained from the CITS results for a defect-free area of the $\mathrm{Ge}(111) 2 \times 1$ surface. The large peak around $0.19 \mathrm{eV}$ can be assigned to the onset of the unoccupied surface states conduction band $(\mathrm{CB}) \pi_{\mathrm{CB}}^{*}$ of the quasi-1D (weakly coupled) $\pi$-bonded rows of the $\mathrm{Ge}(111) 2 \times 1$ surface. $^{23,24}$ Furthermore, two energy gaps can be clearly discerned: A narrow energy gap of about $0.17 \mathrm{eV}$ and a wide energy gap $E_{g}$ of about $0.74 \mathrm{eV}$. The latter should correspond to the forbidden energy gap of the projected bulk band structure of the Ge(111) surface at low temperature. The narrow gap can be linked to the energy gap between the filled bulk valence band (VB) and the unoccupied surface states conduction band $\pi_{\mathrm{CB}}^{*}$. It is well known that, independent of the type of doping (n-type or $p$-type) and of the doping concentration, the Fermi level $E_{F}$ of the Ge(111) surface is pinned by surface states that exist near the top of the VB. ${ }^{25-28}$ The exact value of the energy difference between the top of the bulk VB and the bottom of the surface-states conduction band $\pi_{\mathrm{CB}}^{*}$ is, however, not well known. Since the 
doping level of our sample is rather low $\left(\simeq 2 \times 10^{16} \mathrm{~cm}^{-3}\right)$, it is reasonable to assume that the Fermi level $E_{F}$ is not inside the surface-states conduction band $\pi_{\mathrm{CB}}^{*}$, but rather located at the top of the bulk VB. ${ }^{11}$ We therefore conclude that the narrow gap indeed corresponds to the energy gap between the top of the bulk VB and the bottom of the surface-states conduction band $\pi_{\mathrm{CB}}^{*}$.

The position of the different energy bands observed on the $\mathrm{Ge}(111) 2 \times 1$ surface is represented by the gray rectangles at the bottom of Fig. 4. From the normalized conductance spectrum it can be concluded that the energy bands of the (111) oriented surface of $p$-type Ge are nearly flat. The width of the bonding $\left(\pi_{\mathrm{VB}}\right)$ and the antibonding $\left(\pi_{\mathrm{CB}}^{*}\right)$ surface-states bands have already been investigated both theoretically 7,23 and experimentally ${ }^{28}$ and are 0.8 and $1.2 \mathrm{eV}$ for $\pi_{\mathrm{VB}}$ and $\pi_{\mathrm{CB}}^{*}$, respectively. By means of STM Feenstra et al. ${ }^{6}$ have determined the gap between these bands to be around $0.54 \pm 0.04 \mathrm{eV}$, a value that is consistent with the results of photoemission and inverse photoemission experiments. For $p$-type Ge the unoccupied surface states are the only states that are available for tunneling within the bulk band gap region: there is no overlap of the $\pi_{\mathrm{CB}}^{*}$ surface-states conduction band with the bulk $\mathrm{CB}$, implying that a correct description of the tunneling processes in such systems requires that a relatively low relaxation rate from the surface towards the bulk states has to be considered. The situation strongly differs for heavily doped $n$-type Ge, where band bending almost exceeds the band-gap value. ${ }^{19}$

Coming back to the main panel of Fig. 4, we note that the slope of the $d I / d V(V)$ spectra II and III (recorded on the DB) near the lower edge of the surface conduction band $\pi_{\mathrm{CB}}^{*}$ is clearly different from the slope of spectrum I [recorded on the surrounding defect-free $\mathrm{Ge}(111) 2 \times 1$ surface]. For spectrum I there occurs a quite steep transition from zero conductance inside the surface band gap towards high conductance inside the surface-states conduction band $\pi_{\mathrm{CB}}^{*}$. The different slope indicates that, although surface states remain present at the DB, they appear to be strongly perturbed. For spectrum I the high conductance is reached at a tunneling voltage that within the experimental error corresponds to the energy threshold energy $E_{\text {on }}$ above which spatial oscillations of the LDOS can be observed.

\section{DISCUSSION AND COMPARISON WITH THEORETICAL MODEL CALCULATIONS}

In this section we present a model that accounts for the main properties of the experimentally observed spatial LDOS oscillations. Figure 5(a) presents a typical LDOS map of spatial LDOS oscillations near a type-B DB on the $\mathrm{Ge}(111) 2 \times 1$ surface. In the inset we present the corresponding $2 \mathrm{D}$ Fourier transform. As discussed above, the atomic corrugation and the spatial LDOS oscillations yield strongly localized maxima in the 2D Fourier transform, implying that these maxima can be considered as "additive contributions" to the LDOS map. Taking the $x$-axis direction along the $\pi$-bonded chains and the $y$ axis orthogonal to the $\pi$-bonded chains, the experimental LDOS $\rho(x, y)$ image in Fig. 5(a) can be described by the following simple expression
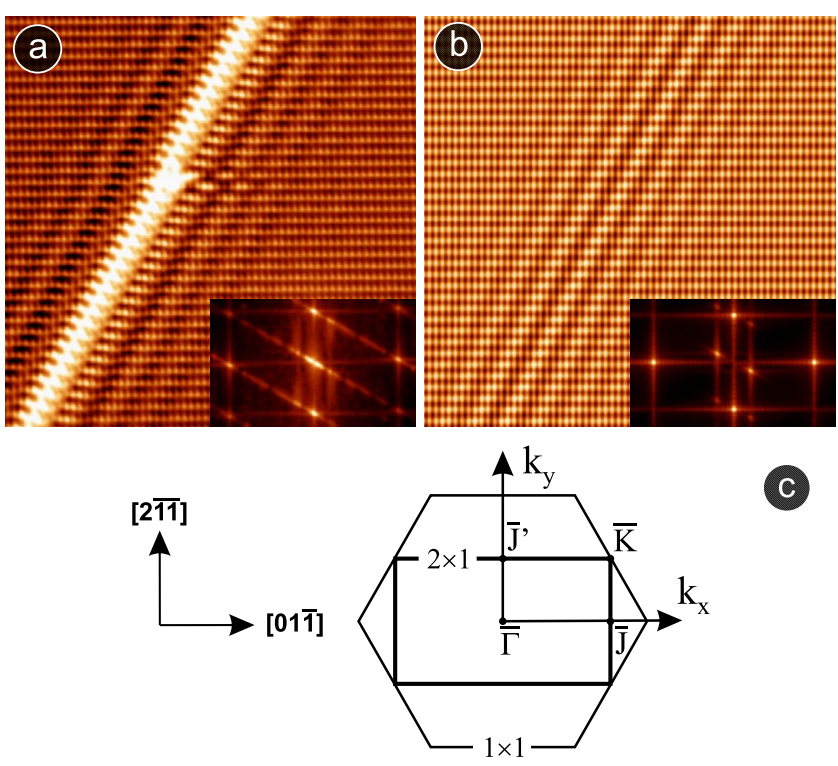

C

FIG. 5. (Color online) (a) Experimental LDOS map of spatial LDOS oscillations at the $\mathrm{Ge}(111) 2 \times 1$ surface $(V=0.61 \mathrm{meV})$ and (b) the corresponding simulated LDOS image. The insets contain the 2D Fourier transform of the LDOS images. (c) Surface Brillouin zones and relevant directions of the $\mathrm{Ge}(111)$ surface.

$$
\begin{aligned}
\rho(x, y)= & A \exp \left(-\frac{2|y-2 x|}{L}\right) \sin \left[\frac{2 \pi(y-2 x)}{\lambda}\right] \\
& + \text { atomic corrugation }+ \text { domain boundary, }
\end{aligned}
$$

where $L$ is the decay length and $\lambda$ is the wavelength of the LDOS oscillations. Figure 5(b) presents a simulated LDOS image based on this expression, showing perfect correspondence to the experimental LDOS map in Fig. 5(a). From a comparison of the 2D Fourier transforms in the insets of Figs. 5(a) and 5(b) it is clear that a plane-wave propagating in the direction normal to the direction of the DB correctly reproduces the 2D Fourier spectrum of the experimental LDOS image.

Our experimental observations can be consistently described by means of a model that takes into account the presence of Tamm surface states. The basic idea is as follows. It is well known that breaking of the three-dimensional (3D) translational symmetry at the surface of a 3D solid can induce the appearance of $p$-orbital-derived surface states that are localized within a narrow surface layer and are commonly referred to as Tamm states. These Tamm states are decaying exponentially into the vacuum while they exhibit an oscillatory decaying behavior into the bulk. While the presence of a 2D surface on a 3D solid results in the appearance of 2D surface states, a 1D surface defect (a DB in our case) on the $2 \mathrm{D}$ surface in a similar way causes the appearance of $1 \mathrm{D}$ surface states.

The Ge(111) $2 \times 1$ surface consists of $\pi$-bonded chains of Ge atoms. The surface unit cell contains two inequivalent atoms, one of them being shifted towards the vacuum (the $u p$ atom), the other being shifted towards the bulk (the down atom). Each up/down surface atom has one dangling-bond $p$ orbital and significant charge transfer occurs from the orbital 
of the down atom to the orbital of the up atom. This causes the appearance of two surface-states bands. The bonding surface-states band $\pi_{\mathrm{VB}}$, which is derived from the up atom orbital, is filled, while the antibonding surface-states band $\pi_{\mathrm{CB}}^{*}$, which is derived from the down atom orbital, is empty. Tilting of the up-down dimer bond gives rise to an energy gap between the $\pi_{\mathrm{VB}}$ and $\pi_{\mathrm{CB}}^{*}$ surface-states bands. ${ }^{7,23}$ The localization of dangling bonds on the up/down atoms in $\pi$-bonded chains gives rise to the quasi-1D character of the surface states, resulting in a strong dispersion along $\pi$-bonded chains in the $[01 \overline{1}]$ direction $(\bar{\Gamma}-\bar{J}$ direction in the surface Brillouin zone) and a weak dispersion in the perpendicular $[2 \overline{11}]$ direction $(\bar{J}-\bar{K}$ direction in the surface Brillouin zone). ${ }^{11,29,30}$ On the other hand, the $\pi_{\mathrm{VB}}$ and $\pi_{\mathrm{CB}}^{*}$ surface states cannot be treated as being perfectly $1 \mathrm{D}$ since the interaction between $\pi$-bonded chains is not negligibly small. The latter interaction has important consequences. Strictly speaking, a $1 \mathrm{D}$ defect on a $2 \mathrm{D}$ surface should result in a $1 \mathrm{D}$ character of the derived surface states. However, when the interaction between $\pi$-bonded chains is taken into account, pure 1D surface states are transformed into strongly anisotropic but nevertheless 2D states. This is indeed confirmed by our experiments. From Figs. 1 and 5 it is clear that the wave vector of the spatial LDOS oscillations is not parallel to the $\pi$-bonded chains. Consequently, the oscillations need to be treated as a quasi-two-dimensional phenomenon.

In order to achieve a quantitative description of the experimental observations we performed analytical calculations within a tight-binding approach. The Ge(111) $2 \times 1$ surface is modeled by linear atomic chains, consisting of two inequivalent atoms $1 / 2$ with energy levels $\varepsilon_{1} / \varepsilon_{2}$ and tunneling matrix elements $T(1 \rightarrow 2$ tunneling $)$ and $t(2 \rightarrow 1$ tunneling $)$. This is a reasonable assumption if one bears in mind the fact that the $\mathrm{Ge}(111) 2 \times 1$ surface consists of up/down atom dimers. The unperturbed energy levels $\varepsilon_{1}$ and $\varepsilon_{2}$ reside in the filled $\pi_{\mathrm{VB}}$ and empty $\pi_{\mathrm{CB}}^{*}$ surface-state band, respectively. The interaction between atomic chains is described in terms of a tunneling amplitude $\tau$, which has the same value for all atoms in the chain. The potential introduced by the DB is modeled in terms of a hard wall potential with height $\tilde{W}$. All possible relaxation processes are described using one single parameter $\gamma$. The LDOS can be obtained from a formalism based on the Green's-functions diagram technique. First, we calculate the LDOS for a single atomic chain. Next, we use the calculated LDOS for a single atomic chain to add the influence of the interchain interaction $\tau$ and of the relaxation $\gamma$. Finally, we add the scattering at the DB, which is rotated over a certain angle with respect to the atomic chain direction.

In the case of an isolated chain $(\tau=0)$ the Green's functions can be obtained from the Dyson equations in Fourier space

$$
\begin{aligned}
& G_{11}^{R}\left(\omega, \kappa_{x}\right)=G_{11}^{0 R}(\omega)+G_{11}^{0 R}(\omega) \xi\left(\kappa_{x}\right) G_{21}^{R}\left(\omega, \kappa_{x}\right), \\
& G_{21}^{R}\left(\omega, \kappa_{x}\right)=G_{22}^{0 R}(\omega)+G_{22}^{0 R}(\omega) \xi^{*}\left(\kappa_{x}\right) G_{11}^{R}\left(\omega, \kappa_{x}\right),
\end{aligned}
$$

where

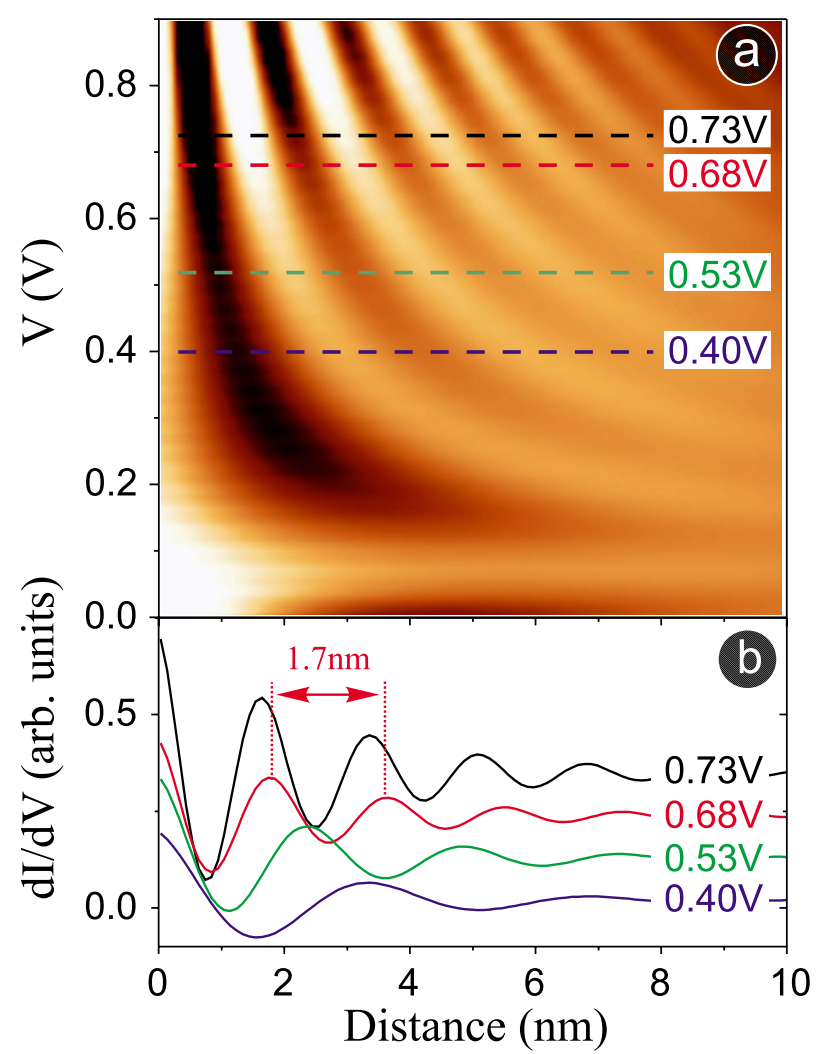

FIG. 6. (Color online) (a) Calculated 2D map of the spatial LDOS oscillations near a DB, revealing multiple hyperboliclike branches. (b) Cross sections of the color map in (a) at values of tunneling voltage indicated in (a).

$$
\begin{gathered}
G_{11}^{0 R}=\frac{1}{\omega-\varepsilon_{1}} \quad \xi\left(\kappa_{x}\right)=\sum t e^{i \kappa_{x} a}+T e^{-i \kappa_{x} a}, \\
G_{22}^{0 R}=\frac{1}{\omega-\varepsilon_{2}} \quad \xi^{*}\left(\kappa_{x}\right)=\sum t e^{-i \kappa_{x} a}+T e^{i \kappa_{x} a} .
\end{gathered}
$$

Here, $a$ is the distance between equivalent atoms along the chain. The Green's functions can be rewritten as

$$
\begin{aligned}
& G_{11}^{R}\left(\omega, \kappa_{x}\right)=\frac{G_{22}^{0 R-1}(\omega)-\xi\left(\kappa_{x}\right)}{G_{11}^{0 R-1}(\omega) G_{22}^{0 R-1}(\omega)-\varepsilon\left(\kappa_{x}\right)}, \\
& G_{22}^{R}\left(\omega, \kappa_{x}\right)=\frac{G_{11}^{0 R-1}(\omega)-\xi^{*}\left(\kappa_{x}\right)}{G_{11}^{0 R-1}(\omega) G_{22}^{0 R-1}(\omega)-\varepsilon\left(\kappa_{x}\right)},
\end{aligned}
$$

where $\varepsilon\left(\kappa_{x}\right)=\left|\xi\left(\kappa_{x}\right)\right|^{2}=t^{2}+T^{2}+2 t T \cos \left(2 \kappa_{x} a\right)$.

When the interaction $\tau$ between atomic chains as well as the relaxation $\gamma$ are taken into account, the Green's functions depend on both spatial components of the wave vector, i.e., $\kappa_{x}$ and $\kappa_{y}$. The Green's functions can again be obtained from the Dyson equations [see Eq. (1)] with the functions $G_{11}^{R}\left(\omega, \kappa_{x}\right)$ and $G_{22}^{R}\left(\omega, \kappa_{x}\right)$ now taking into account interchain interaction $\tau$ and relaxation $\gamma$. The Green's functions then become 


$$
\begin{aligned}
& G_{11}^{R}\left(\omega, \kappa_{x}, \kappa_{y}\right)=\frac{\omega-\varepsilon_{2}-\xi\left(\kappa_{x}\right)}{\left\{\left(\omega-\varepsilon_{2}+i \delta\right)\left(\omega-\varepsilon_{1}+i \delta\right)-\varepsilon\left(\kappa_{x}\right)-\left[\omega-\varepsilon_{2}-\xi\left(\kappa_{x}\right)\right] 2 \tau \cos \kappa_{y} b+i \gamma\right\}}, \\
& G_{22}^{R}\left(\omega, \kappa_{x}, \kappa_{y}\right)=\frac{\omega-\varepsilon_{1}-\xi^{*}\left(\kappa_{x}\right)}{\left\{\left(\omega-\varepsilon_{2}+i \delta\right)\left(\omega-\varepsilon_{1}+i \delta\right)-\varepsilon\left(\kappa_{x}\right)-\left[\omega-\varepsilon_{2}-\xi\left(\kappa_{x}\right)\right] 2 \tau \cos \kappa_{y} b+i \gamma\right\}},
\end{aligned}
$$

where $\xi\left(\kappa_{x}\right)=\left(T e^{2 i \kappa_{x} a}+t\right) / e^{i \kappa_{x} a}$ and $b$ is the distance between equivalent atoms in neighboring chains. As indicated above, the parameter $\gamma$ takes into account all possible processes responsible for the damping of the spatial LDOS oscillations, including electron-electron and electron-phonon interaction. The physical mechanisms causing inelastic scattering have already been discussed in detail for the case of standing- wave patterns in the LDOS of metal surfaces observed with STM. ${ }^{31}$ As demonstrated in Sec. III C, decay of the LDOS oscillation patterns due to the finite temperature and modulation voltage amplitude for the lock-in amplifier based detection can be ruled out as the dominating mechanism behind the experimentally observed decay.

The band gap $E_{g s}$ for the surface states is given by

$$
E_{g s}=-\tau+\frac{1}{2} \sqrt{\left(\varepsilon_{1}-\varepsilon_{2}\right)^{2}+4(t-T)^{2}+4 \tau\left[\varepsilon_{1}-\varepsilon_{2}+\tau+2(t-T)\right]} .
$$

From Eq. (2) we conclude at this stage that, except for the very special case when $\varepsilon_{1}=\varepsilon_{2}$ and $T=t=\tau$, two separate bands with a gap in between are present in the energy spectrum of the surface states.

Next, we include the influence of the DB into our model that already takes into account interchain interaction and relaxation. As mentioned before, the DBs on the $\mathrm{Ge}(111) 2 \times 1$ surface are rotated over an angle $\pm \pi / 6$ with respect to the direction of the $\pi$-bonded chains. In order to simplify the analysis, the coordinate system for our model calculations is rotated from the position where the $x$ axis coincides with the direction of the $\pi$-bonded chains $(x, y)$ to the position where the $y$ axis coincides with the direction of the DB $\left(x_{1}, y_{1}\right)$. Due to the rotation of the coordinate system the DB potential and the Green's functions depend on both the wave vectors in the original $\left[\vec{\kappa}=\left(\kappa_{x}, \kappa_{y}\right)\right]$ and the rotated $\left[\overrightarrow{\kappa_{1}}=\left(\kappa_{x 1}, \kappa_{y 1}\right)\right]$ coordinate system.

The Green's functions for the surface states in the presence of the DB potential can be written in matrix form

$$
\begin{aligned}
\hat{G}^{R}\left(\omega, \vec{\kappa}, \vec{\kappa}_{1}\right)= & \hat{G}^{0 R}\left(\omega, \vec{\kappa}, \vec{\kappa}_{1}\right) \\
& +\sum_{\kappa_{x 2}, \kappa_{y 2}} \hat{G}^{0 R}\left(\omega, \vec{\kappa}, \overrightarrow{\kappa_{1}}\right) \hat{W} \hat{G}^{R}\left(\omega, \overrightarrow{\kappa_{1}}, \overrightarrow{\kappa_{2}}\right),
\end{aligned}
$$

where

$$
\hat{G}^{0 R}\left(\omega, \vec{\kappa}, \vec{\kappa}_{1}\right)=\left(\begin{array}{ll}
G_{11}^{0 R}\left(\omega, \vec{\kappa}, \vec{\kappa}_{1}\right) & G_{12}^{0 R}\left(\omega, \vec{\kappa}, \vec{\kappa}_{1}\right) \\
G_{21}^{0 R}\left(\omega, \vec{\kappa}, \vec{\kappa}_{1}\right) & G_{22}^{0 R}\left(\omega, \vec{\kappa}, \vec{\kappa}_{1}\right)
\end{array}\right),
$$

$$
\hat{W}=\left(\begin{array}{cc}
\tilde{W} & 0 \\
0 & \widetilde{W}
\end{array}\right)
$$

and

$$
\hat{G}^{R}\left(\omega, \vec{\kappa}, \vec{\kappa}_{1}\right)=\left(\begin{array}{ll}
G_{11}^{R}\left(\omega, \vec{\kappa}, \vec{\kappa}_{1}\right) & G_{12}^{R}\left(\omega, \vec{\kappa}, \vec{\kappa}_{1}\right) \\
G_{21}^{R}\left(\omega, \vec{\kappa}, \vec{\kappa}_{1}\right) & G_{22}^{R}\left(\omega, \vec{\kappa}, \vec{\kappa}_{1}\right)
\end{array}\right) .
$$

$\overrightarrow{\kappa_{2}}=\left(\kappa_{x 2}, \kappa_{y 2}\right)$ is a summation index in the Dyson equations, Eq. (3). The Green's function $G_{11}^{0 R}\left(\omega, \vec{\kappa}, \vec{\kappa}_{1}\right)$ is equal to the function $G_{11}^{R}\left(\omega, \kappa_{x}, \kappa_{y}\right)$, while the Green's function $G_{22}^{0 R}\left(\omega, \vec{\kappa}, \vec{\kappa}_{1}\right)$ is equal to $G_{22}^{R}\left(\omega, \kappa_{x}, \kappa_{y}\right)$, which takes into account the interaction between atomic chains in the absence of a DB. The denominator of the final expression for the full Green's functions $G_{i 1, j 2}^{R}\left(\omega, \kappa_{x}, \kappa_{y}, \kappa_{x 1}, \kappa_{y 1}\right)$ provides us with the energy spectrum of the surface localized Tamm states. For simplicity we consider an infinitely large potential $(\tilde{W} \rightarrow \infty)$.

The dependence of the LDOS of the surface states on the distance in a direction perpendicular to the DB is given by

$$
\rho(x, y)=-\frac{1}{\pi}\left[\operatorname{Im} \sum_{\vec{\kappa}, \vec{\kappa}_{1}} \hat{G}^{R}\left(\omega, \vec{\kappa}, \vec{\kappa}_{1}\right) e^{i \kappa_{x} x} e^{-i \kappa_{1 y} y}\right] .
$$

The results of numerical calculations based on Eq. (4) are presented in Fig. 6. The color map in (a) gives the 2D spatial variation in the local density of surface states $\rho$ in the vicinity of a DB. The abscissa corresponds to the distance from the DB while the ordinate corresponds to the value of tunneling voltage. In panel (b) of Fig. 6 we illustrate the 1D variation in the LDOS of the surface states versus distance 
from the $\mathrm{DB}$ in the direction perpendicular to the $\mathrm{DB}$ at the tunneling voltages indicated in both (a) and (b). The 1D graphs in Fig. 6(b) correspond to cross sections of the 2D LDOS at the selected tunneling voltages that are indicated by the horizontal colored dashed lines in Fig. 6. The lowfrequency background has been filtered out from the cross sections. The parameters used for the model calculations in Fig. 6(b) are $a=4 \AA$ and $b=6.9 \AA$. For the other parameters we use $t=1.5 \mathrm{eV}, T=1.2 \mathrm{eV}, \tau=0.25 \mathrm{eV}, \varepsilon_{1}=0.6 \mathrm{eV}$, and $\varepsilon_{2}=-0.2 \mathrm{eV}$.

Using the same values of the model parameters we can also estimate the surface band gap $E_{g s}$, i.e. the energy gap between the occupied $\pi_{\mathrm{VB}}$ and nonoccupied $\pi_{\mathrm{CB}}^{*}$ surfacestate bands (see bottom of Fig. 4). According to Eq. (2) $E_{g s} \simeq 0.57 \mathrm{eV}$. This value nicely agrees with the previously obtained value $E_{g s} \simeq 0.54 \pm 0.04 \mathrm{eV}^{6}$

Important conclusions can be drawn from the results presented in Fig. 6. The calculated LDOS clearly reveals the presence of spatial oscillations. The color map in Fig. 6(a) consists of a few hyperboliclike branches, and the period of the LDOS oscillations decreases when the applied tunneling voltage increases, consistent with our experimental findings in Figs. 3(c) and 3(d). The amplitude of the LDOS oscillations exponentially decreases with increasing distance from the DB, again consistent with the experimental results presented in Fig. 2(b). For the used set of model parameters the theoretically obtained period of the LDOS oscillations of 1.7 $\mathrm{nm}$ at $0.68 \mathrm{~V}$ [see Fig. 6(b)] is in perfect agreement with the experimental value of $1.7 \mathrm{~nm}$ at $0.68 \mathrm{~V}$ [see Fig. 2(b)]. In order to obtain the required agreement between the theoretical and experimental spatial variation in the LDOS, we have to use a relatively large value $\tau=0.25 \mathrm{eV}$ for the interaction between atomic chains. This confirms that the calculation of the spatial LDOS oscillations should be treated as a 2D problem, i.e., the oscillation patterns cannot be simply interpreted in terms of coherent scattering along 1D atomic chains. We assume that the 1D character of the LDOS oscillations around $0 \mathrm{D}$ defects (including individual impurities and DB imperfections) is governed by the same type of physics as the $2 \mathrm{D}$ character of the LDOS oscillations around 1D defects.

In Fig. 7 the experimental and simulated energy dispersion of the LDOS oscillation patterns are compared. The left panel is taken from the experimental data presented in Fig. 3(d). In the right panel we present the calculated energy dispersion of the oscillation patterns, which is obtained by taking the 1D Fourier transform of the simulated energydependent LDOS maps [see Fig. 6(a)], i.e., following a procedure similar to the one that was used to obtain the experimental energy dispersion. The additional experimental data points (colored symbols with error bars) shown in both panels are identical to the data points that were already presented in Fig. 3(d). It can be clearly seen from Fig. 7 that the simulated dispersion perfectly matches the experimental observations for the whole range of tunneling voltages where the LDOS oscillation patterns can be observed. This provides additional proof for the validity of our theoretical model.

Finally, we turn to the influence of relaxation processes. In general, when the damping $\gamma$ of the oscillations is very strong, we should not observe any aligned LDOS oscillation

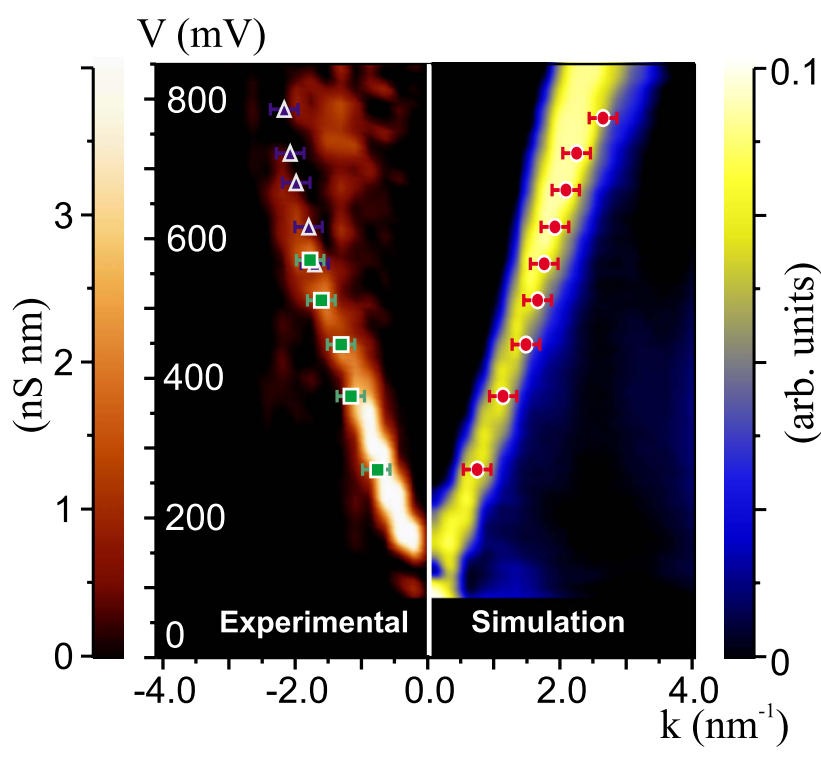

FIG. 7. (Color online) Experimental (left panel) and simulated (right panel) energy versus wave-vector dispersion behavior of the LDOS oscillation patterns. The left panel is taken from Fig. 3(d). The calculated results in the right panel are obtained from 1D Fourier transforms of simulated LDOS maps [see Fig. 6(a)]. The additional experimental data points in both panels (colored symbols with error bars) are taken from Fig. 3(d).

patterns. When the damping $\gamma$ exceeds the interchain interaction $\tau$, the system can be treated as being purely $1 \mathrm{D}$. In the opposite case one needs to take into account the $2 \mathrm{D}$ character of the surface density of states. The ratio $\gamma / \tau$ is determined by the sample surface properties that are affected by scattering at imperfections. The damping is not large in our experiments since the decay length for the spatial LDOS oscillations was found to be $8.7 \mathrm{~nm}$ (see Sec. III B). It is therefore reasonable to assume that in our case $\gamma \ll \tau$. Consequently, the decay of the spatial LDOS oscillations in the direction orthogonal to the DB becomes dominated by the interchain interaction.

\section{CONCLUSIONS}

We investigated into detail the electronic properties of the reconstructed $\mathrm{Ge}(111) 2 \times 1$ surface by means of lowtemperature STM and STS. Spatial oscillations in the local density of electronic states were observed in the vicinity of boundaries between domains with different atomic arrangement within a limited range of tunneling voltages approximately between 0.2 and $0.8 \mathrm{~V}$. Relying on CITS-based measurements of the $I(V)$ curves the energy versus wave-vector dispersion relation was determined. The amplitude of the oscillations was observed to decay exponentially with the distance from the domain boundary. A theoretical model was introduced that is able to explain the behavior of the spatial oscillations in terms of 2D Tamm surface states that are formed within the semiconductor band gap and are scattered at the surface domain boundaries where the $2 \mathrm{D}$ translational 
symmetry of the surface is broken. Our model accurately describes the 2D spatial variation in the local density of states in the vicinity of a domain boundary: The experimentally observed dependence of the oscillation amplitude on the distance from the domain boundary as well as the dependence of the oscillation wavelength on the tunneling voltage are in good agreement with the model-based calculations.

\section{ACKNOWLEDGMENTS}

The research in Moscow has been supported by the RFBR under Grant No. 09-02-01306a and computing facilities of M. V. Lomonosov MSU Research Computing Center. The research in Leuven has been supported by the Fund for Scientific Research-Flanders (Belgium) as well as by the Interuniversity Attraction Poles (IAP) research program.
${ }^{1}$ K. C. Pandey, Phys. Rev. Lett. 47, 1913 (1981).

${ }^{2}$ F. J. Himpsel, P. M. Marcus, R. Tromp, I. P. Batra, M. R. Cook, F. Jona, and H. Liu, Phys. Rev. B 30, 2257 (1984).

${ }^{3}$ R. M. Tromp, R. J. Hamers, and J. E. Demuth, Phys. Rev. B 34, 1388 (1986).

${ }^{4}$ E. S. Hirschorn, D. S. Lin, F. M. Leibsle, A. Samsavar, and T.-C. Chiang, Phys. Rev. B 44, 1403 (1991).

${ }^{5}$ J. A. Kubby and J. J. Boland, Surf. Sci. Rep. 26, 61 (1996).

${ }^{6}$ R. M. Feenstra, G. Meyer, F. Moresco, and K. H. Rieder, Phys. Rev. B 64, 081306(R) (2001).

${ }^{7}$ M. Rohlfing, M. Palummo, G. Onida, and R. DelSole, Phys. Rev. Lett. 85, 5440 (2000).

${ }^{8}$ H. Hirayama, N. Sugihara, and K. Takayanagi, Phys. Rev. B 62, 6900 (2000).

${ }^{9}$ S. Nie and R. M. Feenstra, J. Vac. Sci. Technol. A 22, 1671 (2004).

${ }^{10}$ Y. Einaga, H. Hirayama, and K. Takayanagi, Phys. Rev. B 57, 15567 (1998).

${ }^{11}$ J. M. Nicholls, P. Maartensson, and G. V. Hansson, Phys. Rev. Lett. 54, 2363 (1985).

${ }^{12}$ J. E. Northrup and M. L. Cohen, Phys. Rev. B 27, 6553 (1983).

${ }^{13}$ K. Sagisaka and D. Fujita, Phys. Rev. B 72, 235327 (2005).

${ }^{14}$ K. Nakatsuji, Y. Takagi, and F. Komori, H. Kusuhara, A. Ishii, Phys. Rev. B 72, 241308(R) (2005).

${ }^{15}$ J. V. Barth, H. Brune, G. Ertl, and R. J. Behm, Phys. Rev. B 42, 9307 (1990).

${ }^{16}$ R. Wiesendanger, Scanning Probe Microscopy and Spectroscopy: Methods and Applications (Cambridge University Press, Cambridge, England, 1994).

${ }^{17}$ I. Horcas, R. Fernandez, J. M. Gomez-Rodriguez, J. Colchero, J.
Gomez-Herrero, and A. M. Baro, Rev. Sci. Instrum. 78, 013705 (2007).

${ }^{18}$ D. A. Muzychenko, S. V. Savinov, V. N. Mantsevich, N. S. Maslova, V. I. Panov, K. Schouteden, and C. Van Haesendonck (unpublished).

${ }^{19}$ P. I. Arseyev, N. S. Maslova, V. I. Panov, S. V. Savinov, and C. Van Haesendonck, Pis'ma Zh. Eksp. Teor. Fiz. 82, 312 (2005).

${ }^{20}$ I. Brihuega, P. Mallet, L. Magaud, S. Pons, O. Custance, J. M. Gómez-Rodriguez, and J.-Y. Veuillen, Phys. Rev. B 69, 155407 (2004).

${ }^{21}$ O. Jeandupeux, L. Bürgi, A. Hirstein, H. Brune, and K. Kern, Phys. Rev. B 59, 15926 (1999).

${ }^{22}$ Y. Takagi, K. Nakatsuji, Y. Yoshimoto, and F. Komori, Phys. Rev. B 75, 115304 (2007).

${ }^{23}$ F. Bechstedt, A. A. Stekolnikov, J. Furthmüller, and P. Käckell, Phys. Rev. Lett. 87, 016103 (2001).

${ }^{24}$ K. Kobayashi, Phys. Rev. B 68, 075308 (2003).

${ }^{25}$ D. E. Eastman and W. D. Grobman, Phys. Rev. Lett. 28, 1378 (1972).

${ }^{26}$ D. E. Eastman and J. L. Freeouf, Phys. Rev. Lett. 33, 1601 (1974).

${ }^{27}$ W. D. Grobman, D. E. Eastman, and J. L. Freeouf, Phys. Rev. B 12, 4405 (1975).

${ }^{28}$ J. M. Nicholls and B. Reihl, Surf. Sci. 218, 237 (1989).

${ }^{29}$ J. M. Nicholls, G. V. Hansson, R. I. G. Uhrberg, and S. A. Flodström, Phys. Rev. B 27, 2594 (1983).

${ }^{30}$ R. Haight and M. Baeumler, Phys. Rev. B 46, 1543 (1992).

${ }^{31}$ L. Bürgi, O. Jeandupeux, H. Brune, and K. Kern, Phys. Rev. Lett. 82, 4516 (1999) 